\title{
Cortico-limbic morphology separates tinnitus from tinnitus distress
}

\section{Amber M. Leaver ${ }^{1}$, Anna Seydell-Greenwald ${ }^{1}$, Ted K. Turesky ${ }^{1}$, Susan Morgan ${ }^{2}$, Hung J. Kim ${ }^{3}$ and Josef P. Rauschecker ${ }^{1 *}$}

${ }^{1}$ Department of Neuroscience, Georgetown University Medical Center, Washington, DC, USA

${ }^{2}$ Division of Audiology, Georgetown University Hospital, Washington, DC, USA

${ }^{3}$ Department of Otolaryngology, Georgetown University Hospital, Washington, DC, USA

Edited by:

Jos J. Eggermont, University of

Calgary, Canada

Reviewed by:

Eugen Diesch, CIMH, Germany

Dirk De Ridder, University Hospital

Antwerp, Belgium

*Correspondence:

Josef P. Rauschecker, Department of Neuroscience, Georgetown

University Medical Center, 3970

Reservoir Rd NW, Washington,

DC 20057, USA.

e-mail: rauschej@georgetown.edu
Tinnitus is a common auditory disorder characterized by a chronic ringing or buzzing "in the ear." Despite the auditory-perceptual nature of this disorder, a growing number of studies have reported neuroanatomical differences in tinnitus patients outside the auditory-perceptual system. Some have used this evidence to characterize chronic tinnitus as dysregulation of the auditory system, either resulting from inefficient inhibitory control or through the formation of aversive associations with tinnitus. It remains unclear, however, whether these "non-auditory" anatomical markers of tinnitus are related to the tinnitus signal itself, or merely to negative emotional reactions to tinnitus (i.e., tinnitus distress). In the current study, we used anatomical MRI to identify neural markers of tinnitus, and measured their relationship to a variety of tinnitus characteristics and other factors often linked to tinnitus, such as hearing loss, depression, anxiety, and noise sensitivity. In a new cohort of participants, we confirmed that people with chronic tinnitus exhibit reduced gray matter in ventromedial prefrontal cortex (vmPFC) compared to controls matched for age and hearing loss. This effect was driven by reduced cortical surface area, and was not related to tinnitus distress, symptoms of depression or anxiety, noise sensitivity, or other factors. Instead, tinnitus distress was positively correlated with cortical thickness in the anterior insula in tinnitus patients, while symptoms of anxiety and depression were negatively correlated with cortical thickness in subcallosal anterior cingulate cortex (scACC) across all groups. Tinnitus patients also exhibited increased gyrification of dorsomedial prefrontal cortex (dmPFC), which was more severe in those patients with constant (vs. intermittent) tinnitus awareness. Our data suggest that the neural systems associated with chronic tinnitus are different from those involved in aversive or distressed reactions to tinnitus.

Keywords: tinnitus, anatomical MRI, medial prefrontal cortex, subcallosal anterior cingulate

\section{INTRODUCTION}

Tinnitus pathophysiology is typically thought to involve damage at one or more sites along peripheral and/or central auditory pathways; however, auditory system damage alone does not seem to be sufficient to cause chronic tinnitus. Indeed, a growing body of evidence suggests a relationship between tinnitus and other parts of the brain, both in the form of atypical function and anatomy (Shulman et al., 1995; Lockwood et al., 1998; Mirz et al., 2000; Lowry et al., 2004; Mühlau et al., 2006; Landgrebe et al., 2009; Schlee et al., 2009; Cheung and Larson, 2010; Leaver et al., 2011). Correspondingly, a number of models of tinnitus argue that non-auditory-perceptual networks are necessary components of tinnitus pathophysiology (Jastreboff, 1990; Møller, 2003; Mühlau et al., 2006; Rauschecker et al., 2010; De Ridder et al., 2011).

Of particular relevance is the limbic system, parts of which have been shown to be affected in tinnitus across several studies (Shulman et al., 1995; Lockwood et al., 1998; Mirz et al.,
2000; Lowry et al., 2004; Mühlau et al., 2006; Landgrebe et al., 2009; Cheung and Larson, 2010; Leaver et al., 2011). Our previous work has identified an area of reduced gray matter (GM) in the ventromedial prefrontal cortices ( $\mathrm{vmPFC}$ ) of tinnitus patients using voxel-based morphometry (VBM) (Mühlau et al., 2006; Leaver et al., 2011). Based on these findings, we proposed that chronic tinnitus is caused by failure of the vmPFC-network to suppress aberrant activity in the auditory system (Mühlau et al., 2006; Rauschecker et al., 2010). This hypothesis is consistent with the role the vmPFC and associated structures play in the evaluation of many types of stimuli (Kable and Glimcher, 2009), and characterizes tinnitus as a problem of "noise cancellation."

However, alternative explanations remain. Previous work in mood disorders consistently identifies reduced GM in vmPFC and subcallosal anterior cingulate (scACC) in individuals suffering from clinical depression and anxiety (Drevets et al., 1997; Mayberg, 1997; Koenigs and Grafman, 2009). Also, tinnitus itself can be associated with stress and negative mood (Sullivan et al., 
1988; Folmer et al., 1999; Dobie, 2003). Some theories of tinnitus pathophysiology argue that negative emotional reactions to tinnitus are necessary for the disorder to become chronic (Jastreboff, 1990; De Ridder et al., 2011). If aversive reactions to tinnitus are necessary components of tinnitus pathophysiology, one might expect a positive relationship between the severity of: (1) the auditory-perceptual characteristics of tinnitus, like loudness or awareness (i.e., the amount of time patients are aware of their tinnitus), (2) the patient's suffering and/or the presence of concomitant mood disorders or symptoms, and (3) reductions in vmPFC gray matter or other markers of tinnitus. On the other hand, if vmPFC can affect the gain of tinnitus independent of any emotional reaction (Mühlau et al., 2006; Rauschecker et al., 2010), one would expect reductions in vmPFC gray matter to be related to the auditory-perceptual characteristics of tinnitus, but not its aversiveness or symptoms of depression or anxiety.

Furthermore, due to the inherent limitations of VBM analyses used in previous studies (Mühlau et al., 2006; Landgrebe et al., 2009; Husain et al., 2011; Leaver et al., 2011), the exact nature of this anatomical anomaly in vmPFC and other anatomical markers of tinnitus are unknown. Decreases in cortical thickness, surface area, and gyrification (i.e., curvature or folding) could, in theory, all lead to similar VBM effects (Hutton et al., 2009). In addition, variability in these morphological features across individuals is likely to have different genetic origins and, correspondingly, cellular bases (Panizzon et al., 2009). Thus, going beyond the indirect measures of tissue volume that VBM offers should yield a more detailed picture of tinnitus pathophysiology.

In the current study, we used magnetic resonance imaging (MRI) to identify neuroanatomical correlates of tinnitus, and their relationship to tinnitus characteristics, tinnitus distress, and other factors. In a new cohort of participants, we first measured GM volume using volume-based methods (i.e., VBM). Turning to more sophisticated morphometric techniques, we sought to pinpoint the morphological basis of GM volume reductions using surface-based analyses. Finally, we attempted to determine whether vmPFC morphology reflected tinnitus distress, consistent with the typical view of the limbic system (Jastreboff, 1990; Møller, 2003; De Ridder et al., 2011), or whether vmPFC correlated with auditory-perceptual characteristics of tinnitus, consistent with our model (Mühlau et al., 2006; Rauschecker et al., 2010). By using a series of detailed questionnaires, we also measured the relationship between tinnitus symptoms and non-tinnitus factors like hearing loss, depression, and anxiety.

\section{METHODS \\ PARTICIPANTS}

Forty-four volunteers (23 tinnitus patients, 21 controls) gave informed consent to participate in this study according to procedures of the Institutional Review Board at Georgetown University. Participants were recruited such that the two groups would be matched by age and sex, and standard MRI safety considerations were used as exclusion criteria. Tinnitus patients ranged in age from 23 to 66 years; control participants ranged from 27 to 67 years of age. Detailed characteristics of these groups can be found in Table 1.
Table 1 | Participant characteristics.

\begin{tabular}{lll}
\hline & Patients & Controls \\
\hline $\mathrm{N}$ & 23 & 21 \\
Age (years) & $47.4(2.9)$ & $49.0(2.6)$ \\
Sex (female, male) & 11,12 & 13,8 \\
Mean hearing loss (dB) & $31.1(2.8)$ & $23.4(2.5)$ \\
Loudness discomfort levels (dB)* & $87.1(2.2)$ & $93.7(1.6)$ \\
Noise sensitivity ratings (5-point scale)* & $2.0(0.2)$ & $0.4(0.1)$ \\
Depression score (PHQ9, 27 max score)** & $4.7(1.0)$ & $2.5(0.6)$ \\
Anxiety score (GAD7, 21 max score)** & $4.7(1.0)$ & $2.5(0.7)$ \\
History of mood disorder or medications (yes, no) & 6,17 & 3,18 \\
History of closed-head injury (yes, no) & 4,19 & 3,18 \\
\hline
\end{tabular}

Note: Mean values or counts are given where appropriate; numerical values in parentheses indicate standard error.

* Scores were normalized and combined to form combined Noise Sensitivity Scores.

** Measures were summed to form combined scores.

\section{AUDIOMETRY}

Audiometric testing assessed pure-tone thresholds in all participants at the Division of Audiology at Georgetown University (Figure 1). Pure tones ranging from $250 \mathrm{~Hz}$ to $20 \mathrm{kHz}$ were presented to each ear at increasing intensities until the threshold of detection was reached. The "standard" clinical audiogram tests frequencies from $250 \mathrm{~Hz}$ to $8 \mathrm{kHz}$; we tested additional frequencies above $8 \mathrm{kHz}$ to create an "extended" audiogram for each subject. A conservative "normal" hearing range included thresholds below $20 \mathrm{~dB}$ Hearing Level (HL). Thresholds between 20 and $40 \mathrm{~dB}$ HL were considered a mild loss, 40-60 dB HL was considered moderate, and 60-90 dB HL severe. Thresholds above $90 \mathrm{~dB}$ HL were considered profound. Only two participants (1 tinnitus, 1 control) exhibited profound hearing loss in the standard frequency range (i.e., $\leq 8 \mathrm{kHz}$ ), at a single frequency, $8 \mathrm{kHz}$. Chi-squared analyses indicated no significant difference in the proportion of tinnitus patients and controls with normal, mild, moderate, severe, or profound loss in the standard audiogram $\left(\leq 8 \mathrm{kHz}: X_{(4)}^{2}=6.5, p=0.17\right)$ or extended audiogram $(>8 \mathrm{kHz}$ : $\left.X_{(4)}^{2}=3.98, p=0.41\right)$. Therefore, only the mean hearing loss across the entire audiogram for both ears was considered for further analysis.

Noise sensitivity was tested using Loudness Discomfort Levels (LDLs) and a rating scale included in the Tinnitus Sample Case History Questionnaire (TSCHQ) (Langguth et al., 2007). During LDL assessment, pure tones of various frequencies were presented to both ears with increasing amplitude until the subject indicated an undesirable degree of discomfort. Frequencies tested included a $1 \mathrm{kHz}$ standard and three others dependent on the subject's audiogram. Stimulation was aborted at 100 or $105 \mathrm{~dB}$ HL, so stimuli did not overlap with the entire normal range of LDLs (Morgan et al., 1974). Therefore, LDL scores were combined with a noise sensitivity rating score to yield a combined noise sensitivity measure. Both tinnitus patients and control participants used a 5-point scale to answer the following question from the TSCHQ: Do you have a problem tolerating sounds because they often seem much too loud? That is, do you often experience sounds which other 


\section{A Tinnitus Patients}

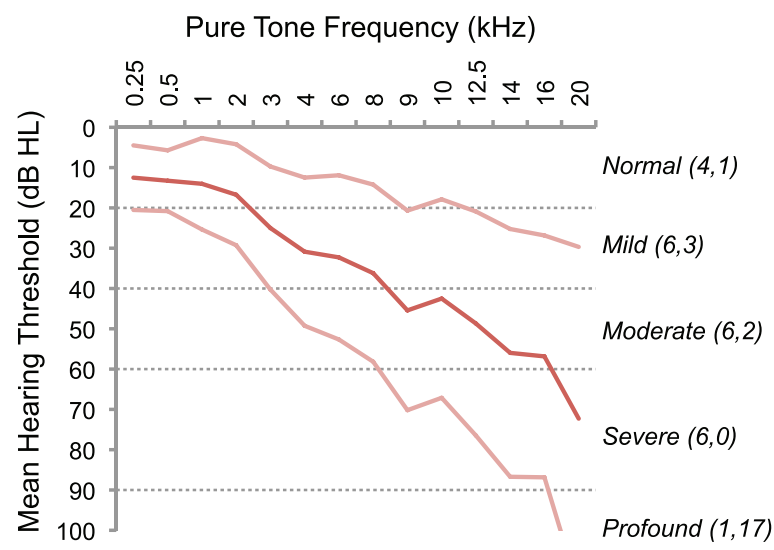

\section{B Control Participants}

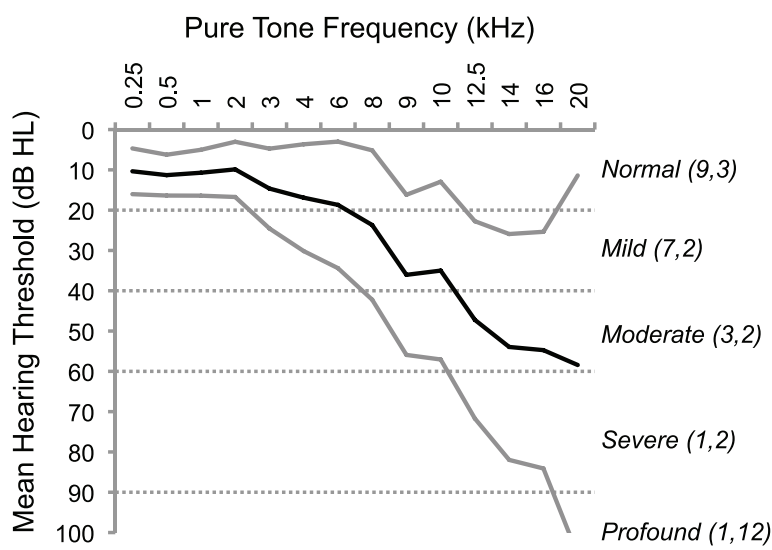

FIGURE 1 | Hearing thresholds in tinnitus patients and controls. The results of pure-tone audiometry are plotted for tinnitus patients $(\mathbf{A})$ and control participants (B). Thresholds in $\mathrm{dB}$ Hearing Level $(\mathrm{HL})$ are displayed on the $y$-axis, and test frequencies are plotted on the x-axis, including standard audiogram frequencies up to $8 \mathrm{kHz}$ and extended test frequencies $>8 \mathrm{kHz}$. Thick lines indicate the mean threshold; thinner lines indicate standard error above and below the mean. Dotted lines delineate ranges of normal ( $\leq 20 \mathrm{~dB} H \mathrm{HL})$, mild (20-40 dB HL), moderate (40-60 dB HL), severe (60-90 dB HL), and profound ( $>90 \mathrm{~dB} H \mathrm{HL}$ ) hearing loss. The number of participants with maximum hearing loss in at least one frequency is indicated for each category in parentheses on the right, separately for standard (first number) and extended (second number) ranges of test frequencies.

people around you find quite comfortable as too loud or hurtful? $(0=$ never $; 1=$ rarely; $2=$ sometimes; $3=$ usually; $4=$ always $)$. To compute the combined score, mean LDLs and noise sensitivity ratings were each normalized (0-1) and summed. Thus, a combined score of 0 indicated minimal noise sensitivity (i.e., LDL score $=100 \mathrm{~dB} \mathrm{HL}$ and rating score of 0 ) and 2 indicated maximal noise sensitivity (i.e., LDL score $=0 \mathrm{~dB} \mathrm{HL}$ and rating score of 4 ).

Tinnitus patients had experienced tinnitus for at least 4 months [mean $(\mathrm{SD})=12.5$ (17.6) years], and reported no history of clinical hyperacusis or phonophobia. Best frequency-match to dominant tinnitus pitch was typically high [mean $(\mathrm{SD})=4,612$
$(3,073) \mathrm{Hz}$, and most reported bilateral tinnitus $(n=18)$. The reported etiology of tinnitus was variable and included change in hearing due to loud noise exposure or other factors $(n=7)$, combined hearing change and head trauma (2), ear or sinus infection (4), unknown or unspecified causes (8), and other factors (2). Tinnitus patients completed two questionnaires related to tinnitus, including the Tinnitus Handicap Inventory (THI) to measure tinnitus impact or distress (Newman et al., 1996) and the TSCHQ to assess tinnitus characteristics. We used three individual questions from the TSCHQ in our analyses. One question measured perceived sensitivity to noise as described above. The other two questions assessed auditory-perceptual characteristics of tinnitus, including the perceived loudness of tinnitus and the percentage of time patients were typically aware of their tinnitus throughout the day (i.e., "awareness"). The latter two questions are worded as follows: (1) Describe the loudness of your tinnitus using a scale from 1 to 100, and (2) What percentage of your total awake time, over the last month, have you been aware of your tinnitus? For example, 100\% would indicate that you were aware of your tinnitus all the time, and 25\% would indicate that you were aware of your tinnitus one fourth of the time.

\section{NEUROLOGICAL HISTORY AND OUESTIONNAIRES}

Participants reported aspects of their medical history during MRI safety screening and the TSCHQ, including history of minor closed-head injury, mood disorder, and current use of neuromodulatory medications. This information is reported in Table 1. A subset of participants reported either a history of mood disorder or current use of neuromodulatory medications (six tinnitus patients, three controls). Of these participants, five reported history of mood disorder (three tinnitus, two control), including depression (two tinnitus, two control), anxiety (two tinnitus), and mild bipolar disorder (one tinnitus). Five participants (four tinnitus, one control) were taking neuromodulatory medication at the time of the study, including GABA agonists or analogues (three patients, one control) and drugs targeting the serotonin system (two patients). These participants were singled out for further analyses.

Participants completed three questionnaires designed to assess symptoms of depression and anxiety: PHQ9 [Patient Health Questionnaire 9, depression; (Kroenke et al., 2001)], GAD7 [Generalized Anxiety Disorder; (Spitzer et al., 2006)], HADS [Hospital Anxiety and Depression Scale (Zigmond and Snaith, 1983)]. The depression and anxiety subscales of the HADS inventory were highly correlated with the PHQ9 and GAD7, respectively $\left(r=0.77, t_{(42)}=7.92, p<0.0001\right.$, and $r=0.85, t_{(42)}=$ 10.33, $p<0.0001)$, and are not discussed further. Depression and anxiety scores were also highly intercorrelated (PHQ9 $\times$ GAD: $\left.r=0.81, t_{(42)}=8.92, p<0.0001\right)$; therefore, PHQ9 and GAD7 measures were summed to create a combined depression and anxiety score for each participant to be used in subsequent analyses.

\section{STATISTICAL ANALYSIS OF AUDIOMETRIC AND QUESTIONNAIRE DATA}

Data acquired during the audiological exam and on questionnaires were analyzed using Matlab's Statistics Toolbox 
(Mathworks). Student's $t$-tests assessed differences between group means on these measures. Equal variance was assumed between groups for those characteristics that were deliberately matched during recruitment (i.e., age), and unequal variance was assumed for all other tests (i.e., mean hearing loss, combined noise sensitivity scores, combined depression and anxiety scores). Chi-squared tests were used to assess differences between groups on categorical data (i.e., sex, history of head injury, history of mood disorder or neuromodulatory medication). Correction for multiple tests was implemented using Bonferroni's method, by dividing alpha by the total number of tests ( $t$ or Chi-squared) performed (7). Relationships between tinnitus symptoms and other patient characteristics were tested using multiple linear regression; no post hoc corrections were applied.

\section{IMAGE ACQUISITION}

Images were acquired using a 3.0 Tesla Siemens TIM Trio scanner. A high-resolution anatomical scan (MPRAGE) was performed for each subject, using a sequence that optimizes image contrast between white and gray matter. MPRAGE parameters were as follows: $\mathrm{TR}=2530 \mathrm{~ms}, \mathrm{TE}=3.5 \mathrm{~ms}$, inversion time $=1100 \mathrm{~ms}$, flip angle $=7^{\circ}, 176$ sagittal slices, matrix size $256 \times 256 \mathrm{~mm}^{2}$, $1 \times 1 \times 1 \mathrm{~mm}^{3}$ resolution.

\section{IMAGE PROCESSING AND ANALYSIS: VOLUME-BASED MORPHOMETRY}

Volumetric analyses were done using VBM in SPM8 (Wellcome Trust Centre for Neuroimaging) using the DARTEL (Diffeomorphic Anatomical Registration Through Exponentiated Lie Algebra; Ashburner, 2007) toolbox. Images were first corrected for inhomogeneities in intensity, and segmented by tissue type using tissue probability templates native to SPM8 (International Consortium for Brain Mapping, www.loni.ucla. edu/ICBM). Study-specific templates were then created using GM images from all subjects. GM images were aligned to this study-specific template, warped to MNI space, and modulated to reflect the amount of deformation applied during normalization (i.e., Jacobian scaling). Images were smoothed with a $6 \mathrm{~mm}^{3}$ FWHM Gaussian kernel, and corrected for total GM volume using proportional scaling. Resulting images were thresholded at 0.20 probability of tissue classification prior to statistical analyses. Between-groups comparisons were conducted voxel-wise across the entire brain, with compensation for unequal variance between groups (i.e., "non-sphericity" correction). Peak MNI coordinates are reported.

Morphometric techniques that rely on tissue segmentation, like VBM, may not be optimal for assessing subcortical structures containing multiple types of tissue (i.e., both white and gray matter) (Ashburner and Friston, 2000). Therefore, additional volumetric analyses of subcortical structures were performed using Freesurfer software version 1.313.2.6 (www.surfer. nmr.mgh.harvard.edu). Images were first corrected for intensity bias and normalized into MNI space. Subcortical structures in these images were identified with an automated procedure that estimates the probability of structure-classification based on prior templates in which those structures were manually identified (Fischl et al., 2002). Subcortical structures identified using this method include the thalamus, caudate, putamen, globus pallidus, nucleus accumbens, amygdala, and hippocampus. Once segmented, the total volume of these structures was calculated in each hemisphere. Because results were similar in each hemisphere, volumes for both hemispheres were summed for statistical analyses reported here. Between-groups differences in total volume were assessed for each structure with Student's $t$-tests assuming unequal variance, using the percentage of total subcortical volume as the dependent measure. Because no tests survived a Bonferroni-correction for the number of $t$-tests performed (7), uncorrected $p$-values are reported.

\section{IMAGE PROCESSING AND ANALYSIS: SURFACE-BASED MORPHOMETRY}

Surface-based analyses were done using Freesurfer. In an automated procedure, images were corrected for intensity bias, and segmented into cortical gray and white matter to reconstruct gray and white matter surfaces. Reconstructed surfaces were inflated, sphere-ized, and aligned with Freesurfer's template average. Automated segmentation of the cortex failed in one tinnitus patient; this participant's data was excluded from surface-based analyses.

For each point (i.e., vertex) on the successfully reconstructed surfaces, values were calculated for pial surface area, thickness, volume (the product of surface area and thickness), and gyrification (i.e., curvature). These data were smoothed in surface-space using a $10 \mathrm{~mm}^{2}$ FWHM Gaussian kernel. A larger kernel is desirable here because smoothing is more likely to occur within tissue type (i.e., gray matter). Between-groups comparisons (i.e., controls $>$ patients) and correlation analyses were conducted vertex-wise across the entire cortex using the general linear model (GLM). Data were not corrected for head size because: (1) significant clusters obtained from the GLM were not correlated with head size and (2) including, e.g., total gray matter volume as a "nuisance" covariate in the GLM did not qualitatively affect the results (data not shown). Peak MNI coordinates are reported.

Region-of-interest (ROI) analyses were also performed. All ROI analyses used the average value (surface area, thickness, or gyrification) for all vertices within the ROI patch as the dependent measure. ROI analyses included $t$-tests (Student's $t$ ) with unequal variance assumed between groups, ANCOVA analyses (group $\times$ relevant covariate), and correlation analyses (Pearson's $r$ ).

In those regions exhibiting reductions in GM volume in tinnitus patients compared to controls in the whole-head, surfacebased analyses, ROI analyses were performed to determine whether volume differences could be explained by differences in surface area, thickness, gyrification, or some combination of these factors. One-tailed $t$-tests were used here, because, for example, a reduction in gray matter in tinnitus patients would be unlikely to be accompanied by increased cortical thickness. Bonferroni-corrections were applied for the total number of ROI tests (12).

To determine whether non-tinnitus factors affected anatomical differences identified between tinnitus patients and controls, we conducted ROI ANCOVAs, using the morphological feature that best described "volume" differences in each ROI 
(surface area, thickness, or gyrification). ANCOVAs allowed us to measure differences between groups while statistically controlling for the effects of three separate covariates: mean hearing loss, combined depression and anxiety scores, or combined noise sensitivity scores. $T$-statistics on the between-groups differences are reported with one-tailed significance values as above, and Bonferroni-adjustments were made for the number of tests in each ROI (3).

ROI analyses also measured the relationship between tinnitus characteristics and cortical morphology in those regions exhibiting significant differences between tinnitus patients and controls. Tinnitus characteristics tested included the number of years since tinnitus onset, tinnitus loudness ratings, the percentage of time patients reported being aware of their tinnitus, and total THI score (i.e., tinnitus distress). One-tailed tests were performed, as we hypothesized that tinnitus patients with more severe symptoms (e.g., louder tinnitus) would exhibit morphology less like controls. Because no tests survived a Bonferroni-correction for the number of correlation tests performed ( 4 per ROI), uncorrected $p$-values are reported.

\section{RESULTS \\ RELATIONSHIPS BETWEEN TINNITUS, TINNITUS SYMPTOMS, AND “NON-TINNITUS" FACTORS}

In order to determine whether chronic tinnitus was concomitant with other non-tinnitus factors, we compared tinnitus patients and controls in a variety of measures (Table 1). Tinnitus patients were deliberately matched by age and sex during recruitment; the groups did not differ on these dimensions (age: $t_{(42)}=$ $0.39, p_{\text {corr }}=1$; sex: $\left.X_{(1)}^{2}=0.35, p_{\text {corr }}=1\right)$. Tinnitus patients also did not differ from controls in mean hearing loss $\left(t_{(42)}=\right.$ $\left.2.11, p_{\text {corr }}=0.14\right)$ or depression and anxiety scores $\left(t_{(35)}=2.02\right.$, $p_{\text {corr }}=0.18$; depression and anxiety scores were highly intercorrelated and thus combined). The proportion of people reporting lifetime incidence of depression, anxiety, or mood-targeting medications did not differ between groups $\left(X_{(1)}^{2}=0.33\right.$, $\left.p_{\text {corr }}=1\right)$, nor did the incidence of head injury differ between groups $\left(X_{(1)}^{2}=0.79, p_{\text {corr }}=1\right)$. Tinnitus patients did score significantly higher in noise sensitivity scores $\left(t_{(35)}=6.26, p_{\text {corr }}=0.04 \times\right.$ $10^{-6}$ ), despite the absence of clinical diagnosis of hyperacusis. Although this indicates a heightened sensitivity to noise on average, many patients overlapped with the range of noise sensitivity scores in controls. Thus, the presence of tinnitus in these patients cannot be fully explained by any of these factors alone.

We also assessed the extent to which the severity of tinnitus can be predicted by other factors, using regression analyses to target two symptoms: tinnitus distress and tinnitus loudness. Tinnitus distress was best predicted by combined depression and anxiety scores $\left(r=0.71, t_{(17)}=3.13, p=0.006\right)$. No other factor explained a significant amount of variance in tinnitus distress, including mean hearing loss $\left(r=0.14, t_{(17)}=1.41, p=0.18\right)$, noise sensitivity $\left(r=0.39, t_{(17)}=-0.43, p=0.67\right)$, percent time aware of tinnitus $\left(r=0.54, t_{(17)}=0.85, p=0.41\right)$, or tinnitus loudness $\left(r=0.31, t_{(17)}=0.40, p=0.70\right)$.

Tinnitus loudness was not significantly related to combined depression and anxiety scores $\left(r=0.23, t_{(17)}=-1.33, p=0.20\right)$ or tinnitus distress $\left(r=0.31, t_{(17)}=0.40, p=0.70\right)$. Instead, the perceived loudness of tinnitus was best predicted by the amount of time patients reported being aware of their tinnitus $(r=0.56$, $\left.t_{(17)}=2.39, p=0.03\right)$, and to a lesser extent noise sensitivity scores $\left(r=0.48, t_{(17)}=2.15, p=0.05\right)$. Degree of hearing loss was also not a good predictor of tinnitus loudness $(r=0.05$, $\left.t_{(17)}=0.53, p=0.60\right)$. These data suggest that the severity of the auditory-perceptual symptoms of tinnitus (e.g., loudness) cannot be explained by ongoing emotional or distressed reactions to tinnitus, and are consistent with previous reports (Hiller and Goebel, 2006).

\section{TINNITUS-RELATED DIFFERENCES IN CORTICAL ANATOMY}

Using a single-voxel DARTEL-VBM analysis across the entire brain, we identified regions with significantly different GM volume in tinnitus patients as compared to controls $(p<0.002$, $k>3.0 \mathrm{~mm}^{3}$; Figure 2). Tinnitus patients exhibited significantly less GM volume in ventromedial prefrontal cortex (vmPFC) compared to control participants (MNI coordinates $X, Y, Z=2,21$, $-15)$. Additional GM reductions were identified in dorsomedial prefrontal cortex (dmPFC; MNI coordinates $X, Y, Z=2,38,39$ ) and left supramarginal gyrus (SMG; MNI coordinates $X, Y, Z=59$, $-40,24)$ adjacent to posterior auditory cortex. Tinnitus patients did not show greater GM volume than controls in any part of the brain at our chosen threshold.

These tinnitus-related reductions in GM volume were confirmed using a different approach targeting the entire cortical
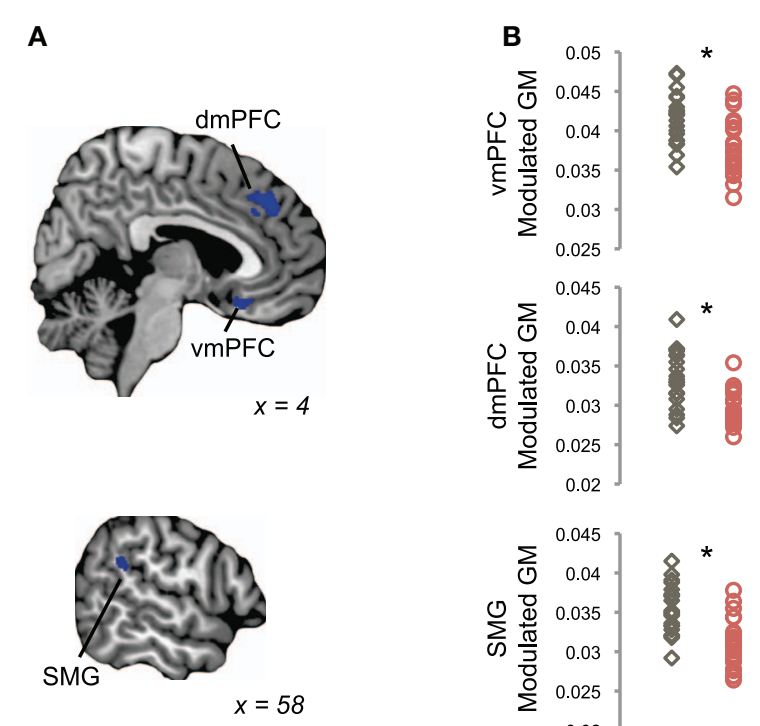

FIGURE 2 | Reductions in gray matter in tinnitus, using a volume-based analysis. (A) Tinnitus patients showed reduced GM volume in ventromedial prefrontal cortex (VmPFC), dorsomedial prefrontal cortex (dmPFC), and supramarginal gyrus (SMG), as compared to control participants in a whole-brain voxel-based morphometry (VBM) analysis (blue). Statistical maps of between-groups differences are shown on parasagittal slices through single-subject template anatomy; the X-dimension in MNI space is displayed for each view. (B) Plots show mean modulated GM values in each cluster shown in (A) for each tinnitus patient (red) and control participant (gray). Asterisks indicate a significant difference between groups, as assessed by the whole-brain analysis in (A). 

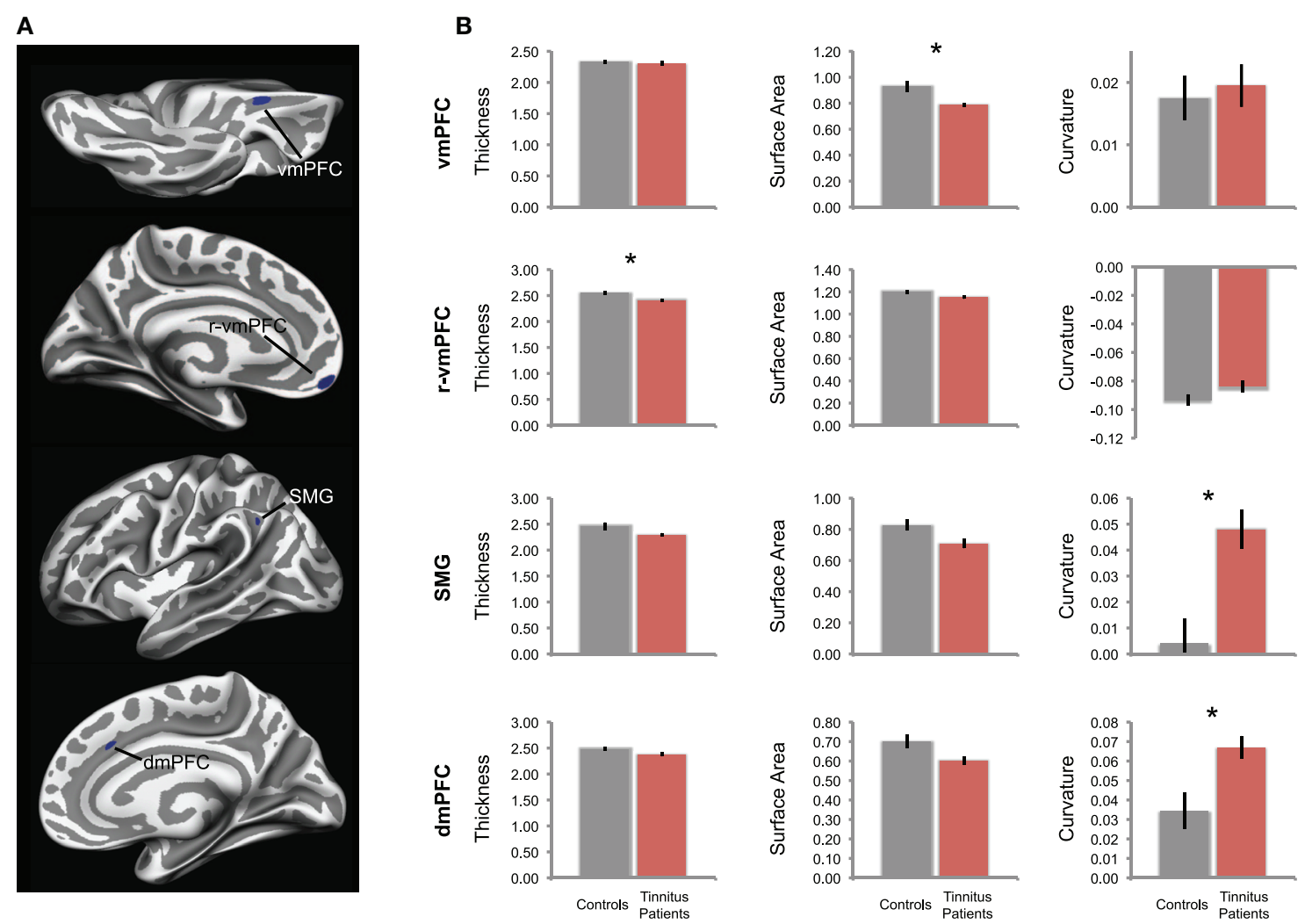

FIGURE 3 | Differences in cortical morphology in tinnitus, in a surface-based analysis. (A) Tinnitus-related reductions in GM volume are confirmed in a single-vertex analysis across the entire cortical surface. Tinnitus patients had significantly smaller GM volumes in the same areas identified in the volume-based analysis shown in Figure 1, vmPFC, dmPFC, and SMG, as well as an additional cluster in rostral vmPFC (r-vmPFC). (B) For each cluster in (A), the mean values of morphological features are plotted, including cortical thickness, surface area, and gyrification (curvature) for each group. For gyrification, positive values indicate sulci, negative values indicate gyri. Tinnitus patients are plotted in red; control participants are plotted in gray. Error bars represent standard error, and asterisks denote significant difference between groups $\left(p_{\text {corr }}<0.05\right)$. surface (i.e., a surface-based analysis in Freesurfer). Again, significantly less GM volume was detected in tinnitus patients in left vmPFC in the olfactory sulcus, right dmPFC in the outer bank of the cingulate sulcus, and left SMG ( $p<0.005$; Figure 3A). An additional left vmPFC region also exhibited less GM volume in tinnitus patients than controls but was located rostral to the one identified during volume-based analysis, which was near the frontal pole ( $\mathrm{r}$-vmPFC).

Reduced GM volume in tinnitus patients could be explained by smaller surface area, thinner cortex, decreased gyrification, or some combination of these factors. Therefore, we used ROI analyses to assess the morphometric origin of volume differences in these four areas (Figure 3B). In posterior vmPFC, volume reductions were best explained by a reduction in cortical surface area $\left(t_{(25)}=3.22, p_{\text {corr }}=0.02\right)$. Cortical thickness and gyrification were not different in this region between groups $\left(t_{(39)}=0.38\right.$, $\left.p_{\text {corr }}=1 ; t_{(41)}=-0.41, p_{\text {corr }}=1\right)$.

In SMG, cortical thickness and surface area did not differ between groups $\left(t_{(28)}=2.00, p_{\text {corr }}=0.33 ; t_{(39)}=2.53, p_{\text {corr }}=\right.$ $0.09)$; instead, gyrification differed between groups $\left(t_{(38)}=\right.$ $\left.-3.61, p_{\text {corr }}=0.005\right)$. Specifically, the sulcus on which the ROI was located was deeper in tinnitus patients than controls (positive values indicate sulci, negative values indicate gyri). Similarly, the volume difference in dmPFC is again best explained by differences in sulcal depth $\left(t_{(34)}=-3.00, p_{\text {corr }}=0.03\right)$; cortical thickness and surface area did not differ in $\operatorname{dmPFC}\left(t_{(40)}=1.85, p_{\text {corr }}=\right.$ $\left.0.43 ; t_{(34)}=2.54, p_{\text {corr }}=0.10\right)$. These differences in gyrification in SMG and dmPFC were associated with a trend toward corresponding reduction in surface area $\left(p_{\text {corr }}=0.09\right.$ and $p_{\text {corr }}=$ 0.10 , respectively), consistent with the idea that deeper sulci typically contain less cortical tissue than other parts of the cortical surface (Fischl and Dale, 2000).

In rostral vmPFC, cortex was thinner in tinnitus patients than in controls $\left(t_{(41)}=2.89, p_{\text {corr }}=0.04\right)$. No significant differences were indicated in gyrification $\left(t_{(41)}=-1.65, p_{\text {corr }}=0.64\right)$ or surface area $\left(t_{(41)}=2.33, p_{\text {corr }}=0.15\right)$ in this region.

\section{TINNITUS-RELATED DIFFERENCES IN SUBCORTICAL ANATOMY}

The volume of subcortical structures, including the thalamus, caudate, putamen, globus pallidus, nucleus accumbens, amygdala, and hippocampus, did not differ between tinnitus patients and controls (Table 2). There was a trend toward increased volume in the left hippocampus of tinnitus patients $\left(t_{(39)}=1.90\right.$, $p=0.07)$, but this difference was not statistically significant. 
Table 2 | Volume of subcortical structures in tinnitus patients and control participants.

\begin{tabular}{llll}
\hline & $\begin{array}{l}\text { Patients } \\
\text { (mean, sd) }\end{array}$ & $\begin{array}{l}\text { Controls } \\
\text { (mean, sd) }\end{array}$ & $\begin{array}{l}\text { Statistics } \\
(\boldsymbol{t}, \boldsymbol{p})\end{array}$ \\
\hline Thalamus & $3.97(0.33)$ & $3.91(0.31)$ & $-0.06,0.54$ \\
Caudate & $2.12(0.23)$ & $2.08(0.23)$ & $-0.07,0.53$ \\
Putamen & $3.03(0.33)$ & $3.01(0.36)$ & $-0.17,0.86$ \\
Globus pallidus & $1.01(0.09)$ & $0.96(0.12)$ & $-1.57,0.13$ \\
Nucleus accumbens & $0.31(0.03)$ & $0.31(0.04)$ & $-0.09,0.93$ \\
Amygdala & $0.87(0.08)$ & $0.85(0.08)$ & $-0.99,0.34$ \\
Hippocampus & $2.23(0.18)$ & $2.18(0.17)$ & $-1.06,0.31$ \\
\hline
\end{tabular}

Note: Volume measures displayed are the percent of total subcortical volume. Statistics reflect the result of $t$ tests, unequal variance assumed.

\section{CORTICAL DIFFERENCES IN TINNITUS AND THEIR RELATIONSHIPS TO NON-TINNITUS FACTORS}

Next, we sought to determine whether non-tinnitus factors affected anatomical differences we identified between tinnitus patients and controls (Figure 4). In the regions of interest (ROIs) defined above, we performed ANCOVAs using the morphological feature that best described "volume" differences in those regions (i.e., surface area for vmPFC, thickness in rostral vmPFC, and gyrification for SMG and dmPFC). Differences were measured between groups while statistically controlling for the potential influence of three separate covariates: mean hearing loss, combined depression and anxiety scores, and combined noise sensitivity scores.

In vmPFC, morphological differences observed between tinnitus patients and controls were not driven by non-tinnitus factors. Differences between these groups in vmPFC were significant when controlling for the influence of hearing loss $\left(t_{(39)}=\right.$ $\left.2.98, p_{\text {corr }}=0.008\right)$, noise sensitivity $\left(t_{(36)}=2.27, p_{\text {corr }}=0.04\right)$, and combined depression and anxiety scores $\left(t_{(39)}=3.21\right.$, $\left.p_{\text {corr }}=0.004\right)$. Differences in surface area in vmPFC are thus likely to be related to the presence of tinnitus, and not other factors.

In $\mathrm{dmPFC}$, there was a non-significant trend toward a difference between groups when controlling for noise sensitivity $\left(t_{(36)}=2.03, p_{\text {corr }}=0.08\right)$. After correcting for an outlier $(>3$ SD below patients' mean), the tinnitus-related difference in morphology was indeed significant $\left(t_{(35)}=2.30, p_{\text {corr }}=0.04\right)$. Hearing loss and depression/anxiety scores did not influence the difference between groups in $\operatorname{dmPFC}\left(t_{(38)}=3.39, p_{\text {corr }}=0.003\right.$ and $t_{(38)}=3.38$, $p_{\text {corr }}=0.003$, respectively).

In SMG and rostral vmPFC, between-groups differences were not driven by hearing loss or depression/anxiety scores, but were influenced by noise sensitivity. In SMG, differences between tinnitus patients and controls were not significant when statistically controlling for noise sensitivity scores $\left(t_{(36)}=1.44\right.$, $\left.p_{\text {corr }}=0.24\right)$. In addition, there was an overall trend toward a positive correlation between noise sensitivity and SMG curvature in both groups $\left(F_{(1,36)}=2.09, p=0.16\right)$. This indicates that the difference in gyrification we identified between tinnitus patients and controls in SMG may be due to differences in noise sensitivity between these participants. In ROI analyses including mean hearing loss and depression/anxiety scores as "nuisance" covariates, between-groups differences were significant $t_{(39)}=3.86, p_{\text {corr }}=0.0006$ and $t_{(39)}=3.12, p_{\text {corr }}=0.005$, respectively).

A similar pattern was observed in rostral vmPFC, in which differences in cortical thickness between tinnitus patients and controls were not significant when taking noise sensitivity scores into account $\left(t_{(36)}=0.76, p_{\text {corr }}=0.68\right)$. Noise sensitivity scores were also modestly correlated with cortical thickness in this region $\left(F_{(1,36)}=2.87, p=0.10\right)$. Differences between groups were significant in analyses controlling for mean hearing loss and depression/anxiety scores in rostral $\operatorname{vmPFC}\left(t_{(39)}=2.57, p_{\text {corr }}=0.02\right.$ and $t_{(39)}=2.57, p_{\text {corr }}=0.02$, respectively).

\section{TINNITUS CHARACTERISTICS AND BRAIN ANATOMY}

The previous analysis identified two areas of the brain with significant anatomical differences between tinnitus patients and controls when controlling for non-tinnitus factors: vmPFC and dmPFC. ROI analyses in these two areas determined whether their morphology related to the severity of tinnitus distress or other symptoms (Figure 5). In dmPFC, a significant positive correlation was present with the percentage of time participants were aware of their tinnitus $(p=0.02$, after removal of outlier described above). Thus, patients with deeper sulci reported being aware of their tinnitus more often than those with dmPFC gyrificiation similar to control participants. A modest negative correlation was also indicated between vmPFC surface area and tinnitus loudness $(p=0.06)$, such that those patients with the highest loudness ratings also exhibited the greatest reductions in cortical surface area. No other correlations were significant $(p>0.05)$.

Correlations between cortical morphology (thickness, surface area, and gyrification) and tinnitus characteristics were also measured across the entire brain $(p<0.0005, k>70$ vertices; Figure 6). Tinnitus distress was positively correlated with cortical thickness in anterior insula (aIns). Percent of time patients reported being aware of their tinnitus positively correlated with STG thickness and SMG surface area. There was an additional area in the postcentral gyrus (PCG) that showed a positive correlation between cortical thickness and duration since the onset of tinnitus. No area in the brain exhibited correlations between tinnitus loudness ratings and whole-brain morphology.

In a post hoc ROI analysis, cortical thickness in aIns was positively correlated with combined depression and anxiety scores in patients $(r=0.78, p<0.0001)$. However, this relationship was not present in controls $(r=-0.16, p=0.50)$.

\section{DEPRESSION AND ANXIETY}

We also assessed the relationship between cortical anatomy and measures of anxiety and depression across the whole brain using the surface-based approach. We looked specifically for negative correlations between depression and anxiety and cortical thickness, in light of previous evidence linking these disorders to cortical volume reductions in scACC. Indeed, we saw a negative correlation between combined depression and anxiety scores (see Methods) and cortical thickness in scACC, when combining data 

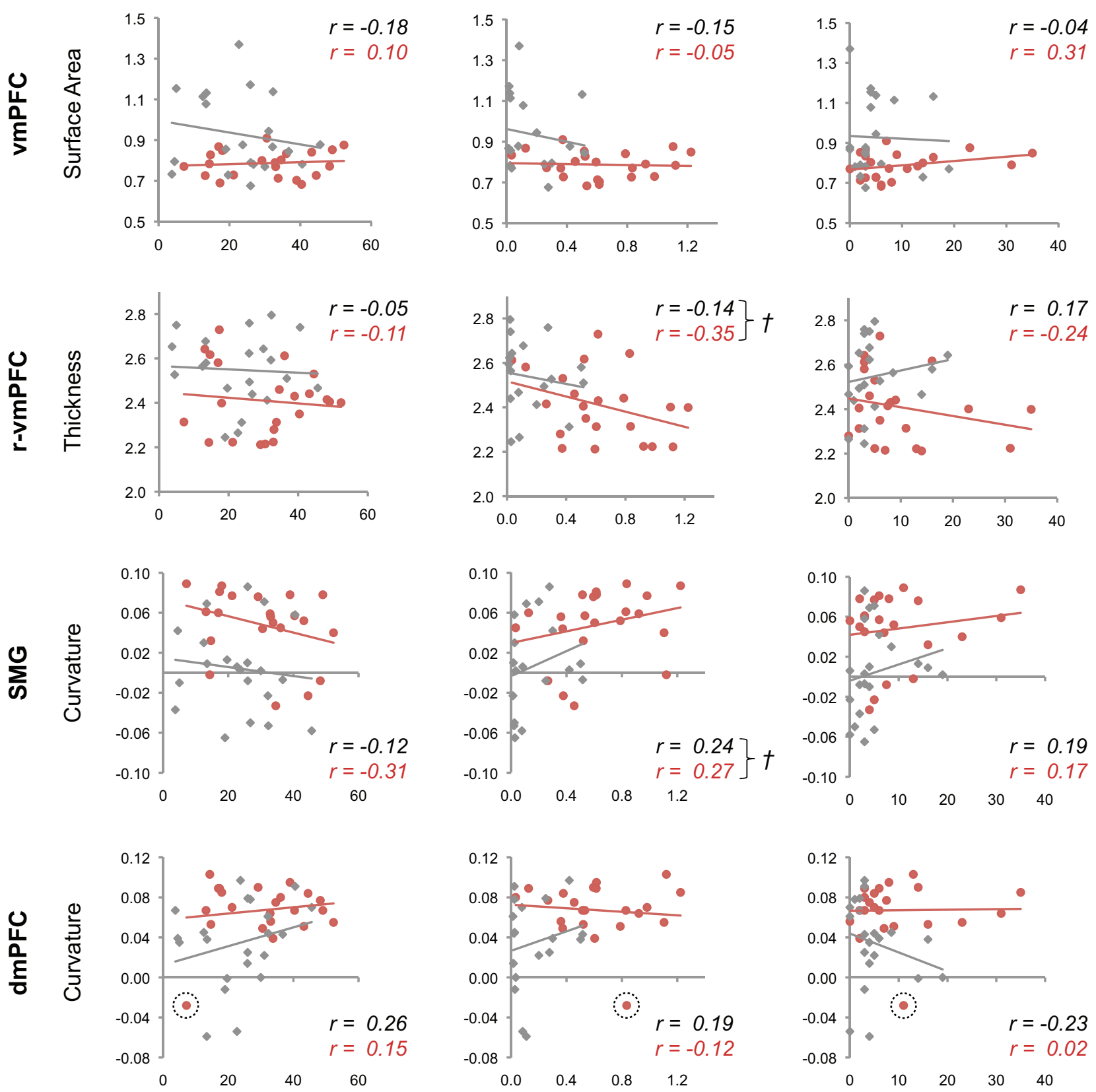

Mean Hearing Loss (dB)

\section{Combined Noise Sensitivity Score}

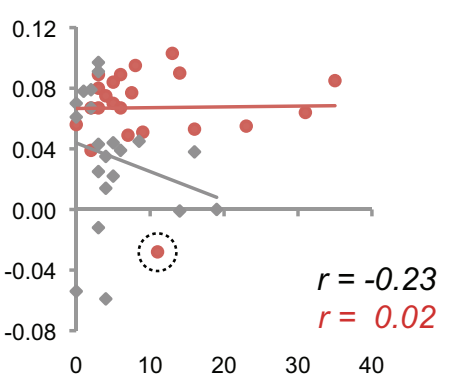

Combined Depression
and Anxiety Score
FIGURE 4 | Tinnitus-related cortical morphology and non-tinnitus factors. Scatterplots show the relationship between morphological features that best explain differences between tinnitus patients and controls in posterior vmPFC, rostral vMPFC, dmPFC, and SMG, and non-tinnitus factors. The mean value is plotted for each tinnitus patient (red) and control (gray) in each cluster, against mean hearing loss (left), noise sensitivity (middle), and combined depression and anxiety scores (right). Regression lines are plotted for each group, and insets display corresponding Pearson's $r$ values for patients (red) and controls (black). Crosses $(t)$ mark those plots for which ANCOVA analysis failed to find a difference between groups, and also indicated moderate correlation between morphological features and non-tinnitus factors. An outlier with dmPFC curvature $>3$

$\mathrm{SD}$ below the mean of tinnitus patients is encircled with a dashed line. from both tinnitus patients and controls $(p<0.005$; Figure 7; MNI coordinates $\left.X, Y, Z=6,24,-8 ; k=12 \mathrm{~mm}^{2}\right)$. An additional cluster was identified in dorsal ACC (MNI coordinates $X, Y, Z=6$, 14,$32 ; k=19 \mathrm{~mm}^{2}$ ).

\section{DISCUSSION}

In our structural MRI study, we identified morphological markers of tinnitus, and assessed the relationship between these markers and various tinnitus characteristics and non-tinnitus factors 

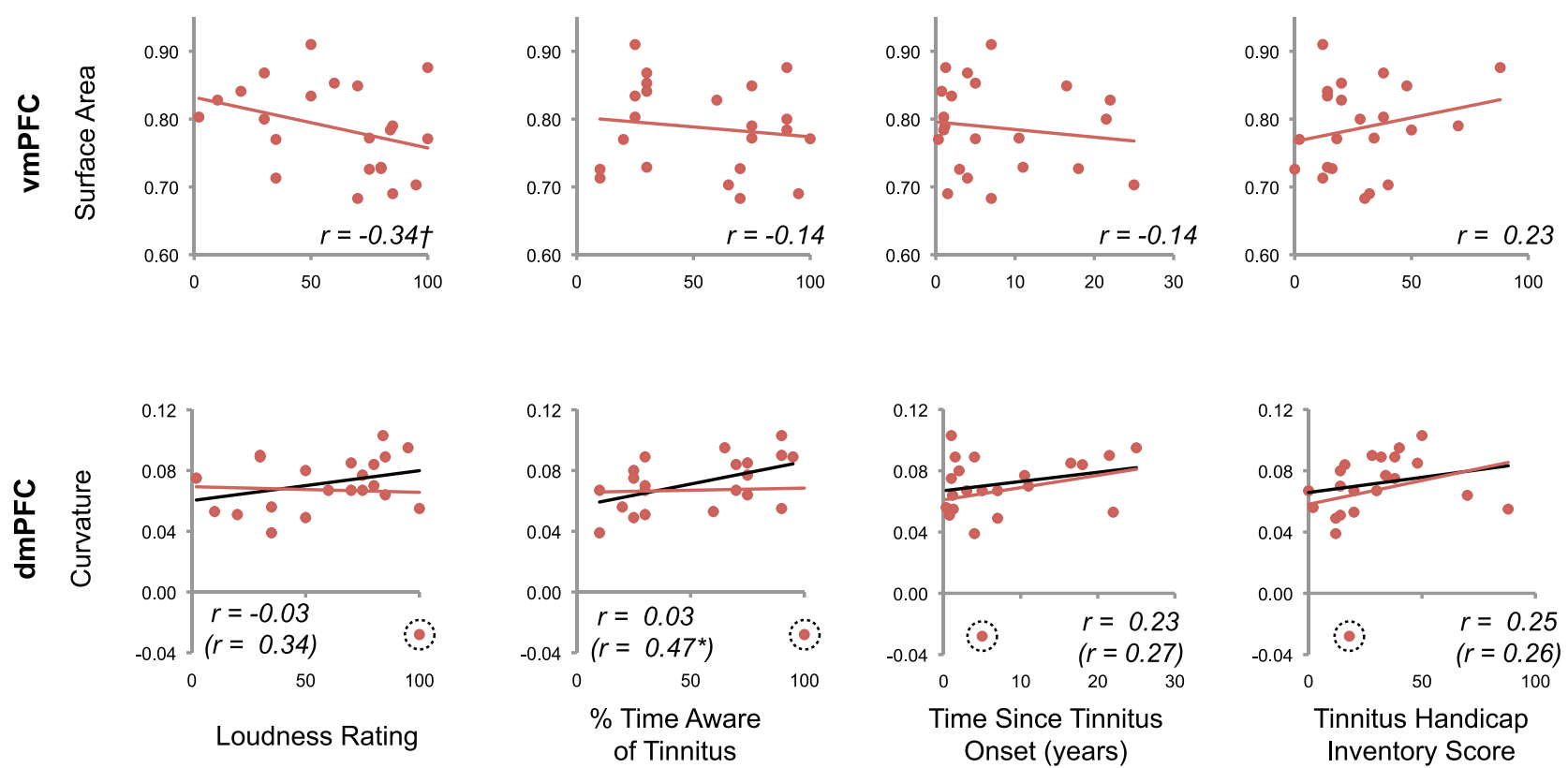

FIGURE 5 | Relationship between tinnitus severity and anatomical markers of tinnitus. For those clusters exhibiting a robust anatomical difference between tinnitus patients and controls, the relationship between morphological features and tinnitus characteristics are shown. Values for each subject are plotted, with corresponding regression lines and Pearson's $r$ values. A modest negative correlation was indicated between vmPFC surface area and tinnitus loudness ( $t p=0.06$, one-tailed test). A significant correlation was also apparent between dmPFC curvature and the percentage of time patients reported being aware of their tinnitus after removal of a statistical outlier ( ${ }^{*} p=0.02$, one-tailed test). Black regression lines and $r$ statistics in parentheses reflect the results of correlation analyses performed after removing this outlier. linked to tinnitus like depression, anxiety, and noise sensitivity. We confirmed the association between tinnitus and reduced GM in vmPFC in an entirely new set of subjects, and identified a new tinnitus-related effect in dmPFC. These morphological differences in vmPFC and dmPFC were not affected by nontinnitus factors or tinnitus distress, but seem to be related to auditory-perceptual characteristics of tinnitus. Tinnitus distress, on the other hand, was linked to variability in cortical thickness in the anterior insula, while depression and anxiety scores predicted cortical thickness in scACC in both tinnitus patients and controls. In addition, we report that noise sensitivity may be related to anatomy in SMG and rostral vmPFC. Taken together, our data suggest that the neural systems related to the tinnitus perception itself are distinct from those affected by tinnitus distress, mood disorders, and noise sensitivity. We take this to mean that aversive or affective reactions to the tinnitus percept may not be necessary for chronic tinnitus to develop, although future research is needed to directly address causality. Regardless, the possibility that the perceptual characteristics of tinnitus are separable from ongoing affective reactions should be considered in current models of tinnitus pathophysiology and in approaches to treatment.

\section{THE ROLE OF MIDLINE FRONTAL CORTEX IN TINNITUS}

In a subregion of vmPFC, we identified GM reductions in a third group of tinnitus patients, providing confirmation of our previous studies (Mühlau et al., 2006; Leaver et al., 2011). We also presented two additional novel findings in the current paper regarding vmPFC morphology. First, we showed that the anatomical anomaly (i.e., GM reduction) in vmPFC of tinnitus patients is due to a reduction in cortical surface area, not cortical thinning or gyrification. Methods used in previous studies have been unable to examine morphology in such detail (Mühlau et al., 2006; Landgrebe et al., 2009; Husain et al., 2011; Leaver et al., 2011; Mahoney et al., 2011). In addition, we demonstrated that reduced gray matter in vmPFC is not correlated with depression, anxiety, or tinnitus distress, suggesting that GM reductions in vmPFC are not likely to be caused by the aversiveness of tinnitus or stress caused by the disorder. Instead, morphology in this area was modestly correlated with the perceptual loudness of the tinnitus sensation, indicating that vmPFC is related to the perception of tinnitus and is thus part of the gating system postulated previously (Rauschecker et al., 2010).

Because the relationship between midline frontal areas and affect is well documented (Drevets et al., 1997; Mayberg, 1997; Koenigs and Grafman, 2009), it was important to show that tinnitus-related effects in vmPFC were not due to, for example, cell death resulting from prolonged exposure to an aversive sound (i.e., tinnitus). If ongoing emotional reactions to tinnitus caused GM loss in vmPFC, one would expect the severity of that loss to correlate with how annoyed or bothered each patient was by his or her tinnitus, or perhaps with the amount of time since the onset of the disorder. We found neither of these to be true. The morphology of this subregion of vmPFC seems to have no bearing on ongoing negative or emotional reactions to tinnitus. 


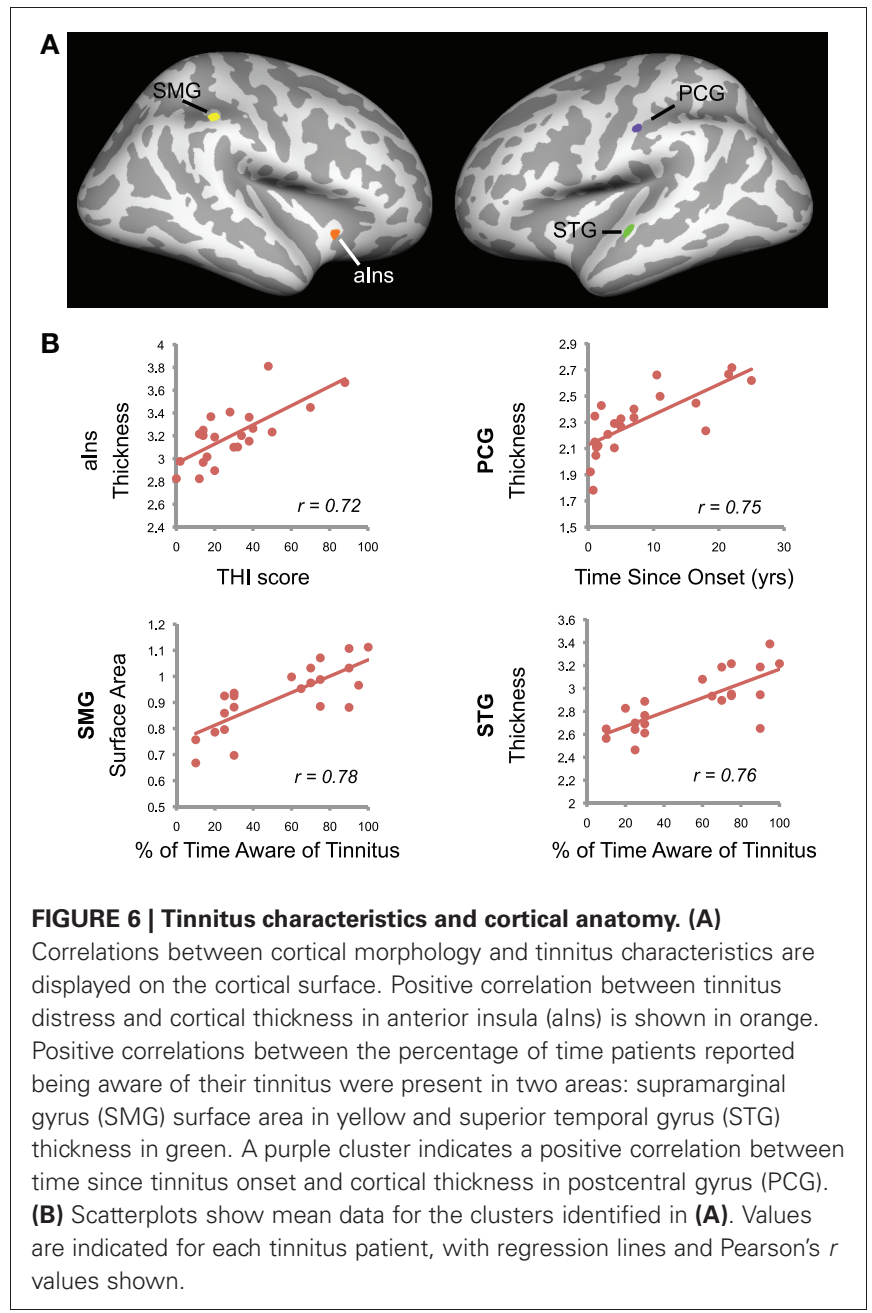

We did identify a relationship between symptoms of anxiety and depression (as measured by questionnaire) and cortical thickness in scACC, close to but clearly separate from tinnitus-related reductions in vmPFC surface area. The scACC has been implicated in mood disorders (Drevets et al., 1997; Mayberg, 1997; Hamani et al., 2011), and is a target of deep brain stimulation treatment for major depression (Lozano et al., 2008). Tinnitus itself is sometimes comorbid with depression (Sullivan et al., 1988; Folmer et al., 1999; Dobie, 2003; Robinson et al., 2008), and even our own behavioral data indicated a (non-significant) tendency for tinnitus patients to score higher on measures of depression and anxiety (uncorrected $p=0.06$; Table 1). However, this correlation between scACC thickness and symptoms of depression and anxiety was present in both tinnitus patients and controls, and there was no difference between groups in cortical thickness in this area. Thus, these data suggest a dissociation between midline frontal areas affected in depression from those affected in tinnitus perception. The extent to which the pathophysiology of depression overlaps with that of anxiety and other mood disorders is an ongoing area of research (e.g., Savitz and Drevets, 2009) and may be informative in future studies of tinnitus and tinnitus-related suffering.
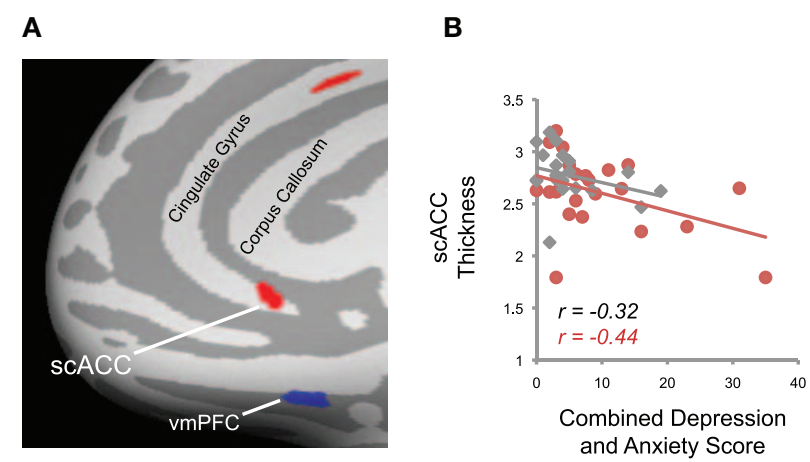

FIGURE 7 | Relationship between depression and anxiety and cortical thickness. (A) Right subcallosal anterior cingulate cortex (scACC) and dorsal anterior cingulate cortex exhibited a negative relationship between combined depression and anxiety scores and cortical thickness (red). The mirrored location of reduced gray matter volume in tinnitus patients in left vmPFC (Figure 3A, top panel) is also displayed for reference (blue), illustrating the spatial dissociation of the two effects. The inflated surface was rotated from the medial aspect of the brain along the $y$-axis so that all clusters are within view. (B) Data from the scACC cluster shown in (A) is plotted for each tinnitus patient (red) and control (gray). Regression lines and Pearson's $r$ values for each group are displayed, which show the negative relationship between depression/anxiety scores and cortical morphology in this region.

Although midline frontal cortex as a whole might be involved in both affective processing and tinnitus (De Ridder et al., 2011), our data indicate a spatial dissociation within this region between tinnitus-related (vmPFC) and mood-related (scACC) effects (Figure 7A). This underscores the importance of appreciating the rich functional heterogeneity within midline frontal cortex (Ongür et al., 2003) when examining the functional and structural effects of tinnitus in this part of the brain. All VBM studies of tinnitus thus far have used comparable imaging parameters, including voxel resolution, and smoothing (Mühlau et al., 2006; Landgrebe et al., 2009; Husain et al., 2011; Leaver et al., 2011; Mahoney et al., 2011); however, most of these studies have not distinguished between tinnitus, tinnitus distress, and negative mood. Our current data indicate that tinnitus research that does not measure symptoms of anxiety and depression as well as tinnitus may run the risk of not unraveling tinnitusrelated effects in vmPFC (Landgrebe et al., 2009; Husain et al., 2011; Mahoney et al., 2011), or conflating the latter with moodrelated effects in scACC (Mühlau et al., 2006; Leaver et al., 2011).

Indeed, we identified an additional midline structure, dmPFC, which showed tinnitus-related effects complementary to those seen in vmPFC. As in vmPFC, morphological differences in dmPFC were not affected by depression, anxiety, or tinnitus distress. Instead, dmPFC morphology, characterized as a difference in cortical curvature, was related to an auditory-perceptual characteristic of tinnitus - awareness, or the proportion of time patients were aware of their tinnitus. This suggests that these two areas may play complementary roles in tinnitus. To the best of our knowledge, these findings in dmPFC are novel, and may result from superior image-alignment afforded by the methods 
used in the current study (i.e., DARTEL-VBM and cortex-based alignment in Freesurfer). Future studies delineating the respective roles of these regions in tinnitus will contribute to the ongoing debate regarding the differential function of dorsal and ventral regions of medial frontal cortex in other domains (Steele and Lawrie, 2004; Etkin et al., 2011; Shackman et al., 2011).

What causes these morphological differences in midline frontal cortex between tinnitus patients and controls remains to be determined. In terms of timing, our data suggest two hypotheses: that the relative size (and cellular make-up) of vmPFC is determined prior to tinnitus onset, or that GM loss in vmPFC occurs soon after the onset of the disorder. In our data, GM levels in vmPFC and dmPFC were the same whether patients had tinnitus for their entire lives or only 4-6 months (Figure 5); longitudinal studies could capture morphological changes over the first few months of tinnitus onset to determine which hypothesis is correct. Apart from timing, there are several possible causes for tinnitus-related differences in vmPFC, which are not mutually exclusive. Genetic factors may determine vmPFC size from birth, or they may cause midline frontal regions to be more vulnerable to stressors or lesions. Environmental factors like chronic stress, depression, or mechanical injuries may also cause cell loss in vmPFC or connected midline structures, or may make the area more susceptible to damage. Indeed, mechanical injuries, such as head trauma or blast injury, can specifically affect ventral frontal regions (Mattson and Levin, 1990; Fujiwara et al., 2008). In theory, damage to a single subregion may also affect other parts of the midline and orbital frontal system, as these areas are richly interconnected (Ongür and Price, 2000). In addition to longitudinal studies to determine timing, histological studies would be useful in determining the cellular bases of tinnitus-related differences in midline frontal cortex. So, although stressful life events or depression may be one way of damaging or over-working this system and causing chronic tinnitus (e.g., combined with sensorineural hearing loss), we argue that it is only one of many ways that the vmPFC-network can be compromised in tinnitus pathophysiology.

Overall, our data are consistent with the idea that midline frontal areas are involved in regulating interoceptive functions, including bodily sensations like pain (Kuchinad et al., 2007), emotions (Drevets et al., 1997; Mayberg, 1997), or even unwanted thoughts or actions (Floresco et al., 2009). In the context of tinnitus, we propose that an "intact" vmPFC is able to suppress aberrant thalamocortical activity in the auditory system (e.g., tinnitus), through its "driver" inputs to inhibitory neurons in the subsection of the reticular nucleus near auditory thalamus (Zikopoulos and Barbas, 2006). If vmPFC is compromised, this circuit is less efficient in regulating unwanted activity, and tinnitus persists (Mühlau et al., 2006; Rauschecker et al., 2010). In the model we propose, a negative or emotional reaction to tinnitus is not necessary for the disorder to become chronic, though stress and negative affect can clearly have modulatory effects on these circuits.

\section{TINNITUS DISTRESS AND CORTICAL MORPHOLOGY}

Tinnitus can be debilitating, but there is also variability in the degree to which tinnitus affects each patient's quality of life
(Heller, 2003; Eggermont and Roberts, 2004). Our behavioral data indicate that the auditory-perceptual symptoms of tinnitus (e.g., perceived loudness or awareness) are not good predictors of tinnitus distress. Correspondingly, those areas of the brain showing tinnitus-related differences in morphology in our study (i.e., vmPFC, dmPFC) do not seem to be affected by tinnitus distress. Instead, a separation seems to exist between those parts of the brain mediating tinnitus, and those meditating the emotional or stress reaction to tinnitus. Specifically, we saw that the severity of tinnitus distress was correlated with cortical thickness in the anterior insula (aIns). Several studies have implicated aIns in pain (DaSilva et al., 2008), stress (Choi et al., 2011; Dannlowski et al., 2012), mood disorders (Mayberg et al., 1999), and tinnitus distress (van der Loo et al., 2011), suggesting it may be involved in mediating the visceral sensations associated with stress and negative affect.

In our data, aIns thickness was also positively correlated with depression and anxiety scores in patients, but not in controls. This may indicate that aIns plays a role in affective reactions specific to tinnitus or other "interoceptive" phenomena (Craig, 2003) not experienced by controls, or that some tinnitus patients process stress or negative affect differently. However, the range of depression and anxiety scores in the current study was limited, and scores for the great majority of participants fell below the cutoff for potential clinical significance. Studies that include a wider range of participants, perhaps including those with clinically diagnosed depression, or who are more severely distressed by their tinnitus, may be better able to address whether some tinnitus patients process stress and negative affect in a unique way.

\section{RELATIONSHIP BETWEEN TINNITUS AND NOISE SENSITIVITY}

Pathological noise sensitivity, or "hyperacusis," is often comorbid with tinnitus (Goldstein and Shulman, 1996; Salvi et al., 2000). Although our participants reported having no history of hyperacusis, our behavioral data support the idea that tinnitus patients tend to be more sensitive to noise overall. The neural basis of clinical hyperacusis is not well understood, but a few human imaging studies report a dissociation between involvement of subcortical auditory structures in hyperacusis and auditory cortex in tinnitus (Gu et al., 2010; Mahoney et al., 2011). Indeed, many studies show auditory cortex hyperactivity in tinnitus (Lockwood et al., 1998; Giraud et al., 1999; Reyes et al., 2002; Plewnia et al., 2007; Leaver et al., 2011), and the current study found a significant relationship between tinnitus awareness and cortical thickness in part of auditory cortex (STG), complementing previous reports of tinnitusrelated GM reductions in auditory cortex (Schneider et al., 2009). These previous studies indicate a separation within the auditory system between sites of tinnitus (cortical) and hyperacusis (subcortical), the latter affecting basic gain-control mechanisms ( $\mathrm{Gu}$ et al., 2010).

With regard to noise sensitivity in our current study, we also found a modest relationship between SMG and rostral vmPFC morphology and combined noise sensitivity scores. Morphology in these regions (curvature in SMG and thickness in r-vmPFC) appeared to differ between groups; however, this effect was actually driven by differences in noise sensitivity scores between 
groups. It is unclear whether these neuroanatomical differences are specific to individuals with comorbid tinnitus and hyperacusis, or whether we might find similar morphological differences in SMG and rostral vmPFC in people with hyperacusis but no tinnitus. Studies specifically designed to address these issues are needed, particularly those including patients with hyperacusis (Gu et al., 2010; Mahoney et al., 2011). Considering the influence of noise sensitivity in tinnitus research (and vice versa) is clearly important.

\section{TINNITUS AND ATTENTION}

The site of tinnitus-related morphological differences we reported in SMG in the current study (MNI coordinates $X, Y, Z=59$, $-40,24)$ is very close to a region of posterior auditory cortex that exhibited hyperactivity in tinnitus patients in our previous study [MNI coordinates of largest cluster $X, Y, Z=56,-40,15$; (Leaver et al., 2011)]. In our previous study, we postulated that this hyperactivity reflected the attempt by patients to separate their tinnitus sound from experimental stimuli in order to complete the task. In everyday situations, the SMG (and other parts of posterior parietal cortex) is involved in the attentional modulation of sensory stimuli (Corbetta and Shulman, 2002, 2011). Adjacent posterior auditory cortex has been shown to be recruited in tasks requiring the separation of competing auditory signals (Zatorre et al., 2002; Alain et al., 2005; Wilson et al., 2007; Wong et al., 2008), often called the "cocktail party problem." Under this assumption, the relationship between right SMG surface area and tinnitus awareness could be explained by inter-individual variability in the capacity to separate meaningful sounds from background noise. In other words, patients with larger (or use-dependent increases in) SMG surface area may be better able to incorporate tinnitus into (relatively) unattended background noise, allowing them to focus on relevant sounds with more success. Indeed, many studies have reported increased activity in posterior auditory cortex in tinnitus (Lockwood et al., 1998; Giraud et al., 1999; Reyes et al., 2002). So, although the role of posterior auditory cortex and adjacent parietal areas as a whole is likely to be more complex (Griffiths and Warren, 2002; Rauschecker and Scott, 2009), these areas are very likely to play a role in the attentional modulation of tinnitus as well.

\section{INTERPRETING OF VARIABILITY IN CORTICAL THICKNESS, SURFACE AREA, AND GYRIFICATION}

Surface-based methods like the ones used here are able to measure morphology in greater detail than volumetric analyses. For example, both surface area and thickness can contribute to the overall volume of cortex, and areas with greater local gyrification are likely to have greater cortical volume (i.e., more gyri $=$ greater surface area $=$ more gray matter). Because these morphological features can have different genetic origins (Panizzon et al., 2009; Winkler et al., 2010; Eyler et al., 2011), precise knowledge of morphological anomalies in clinical populations can increase our understanding of the cellular bases of these anomalies and can serve as a better complement to post mortem histological studies.

However, there is evidence that brain morphology can change with use-dependent experience (May and Gaser, 2006) and age
(Good et al., 2001; Hutton et al., 2009) as well. Thus, neuromorphological effects, as reported here, can have multiple interpretations based on genetics and/or experience. Moreover, it is unclear whether a change in cell number due to experience or cell death would be more likely to affect thickness, surface area, or both. Cortical thickness and surface area both tend to decrease with age (Hutton et al., 2009; Lemaitre et al., 2012), suggesting that atrophy can affect both these neuro-morphological features (even if age is unlikely to play a role in the current study). Indeed, we hypothesized that morphology in some parts of the brain may change progressively in relation to tinnitus onset in the current study; however, we only saw one such effect in an area between the postcentral and angular gyri. This could be evidence of usedependent increases in cell number in this area, though the exact interpretation is unclear. Longitudinal research, both within the context of tinnitus and without, is needed to better understand the effects of plasticity on brain morphology.

\section{CONCLUSIONS: IMPLICATIONS FOR MODELS OF TINNITUS PATHOPHYSIOLOGY}

Overall, the present data support the hypothesis that parts of the limbic system play a central role in tinnitus pathophysiology beyond a mere reaction to the tinnitus sound. Specifically, we argue that chronic tinnitus is caused by failure of the vmPFCnetwork to suppress unwanted activity in the auditory system (Mühlau et al., 2006; Rauschecker et al., 2010). This model is supported by evidence of GM reductions in vmPFC in three independent samples of participants [(Mühlau et al., 2006; Leaver et al., 2011) and the present study], in addition to anomalous activity in this network (Schlee et al., 2009; Leaver et al., 2011; Schecklmann et al., 2011). Furthermore, separate neural systems seem to mediate the tinnitus percept itself and the emotional reaction to tinnitus. Our data showed that the severity of tinnitus distress predicted cortical thickness in aIns, and symptoms of anxiety and depression correlated with scACC thickness; neither of these correlations was present in vmPFC. Note too that morphology in aIns and scACC did not differ between tinnitus patients and controls. Chronic tinnitus may not be caused, as some have argued, by forming negative associations with tinnitus. Stress and negative affect can certainly exacerbate tinnitus (Sullivan et al., 1988; Folmer et al., 1999; Dobie, 2003), but our data suggest that stress and negative affect are not necessary components of chronic tinnitus. The simple fact that not all tinnitus patients are distressed by their tinnitus suggests that models predicated on distress are unlikely to truly capture the disorder. If the neuroanatomical bases of tinnitus-related suffering and negative affect are indeed separable from those mediating the tinnitus signal itself, this underscores the importance of addressing both systems when developing treatment programs for tinnitus (Jastreboff, 2007).

\section{ACKNOWLEDGMENTS}

We wish to thank Sylke-Monina Chowdhury and Brannon Green for their technical assistance. This work was funded by the National Institutes of Health (RC1-DC010720 to Josef P. Rauschecker). 


\section{REFERENCES}

Alain, C., Reinke, K., McDonald, K. L., Chau, W., Tam, F., Pacurar, A., and Graham, S. (2005). Left thalamocortical network implicated in successful speech separation and identification. Neuroimage 26, 592-599.

Ashburner, J. (2007). A fast diffeomorphic image registration algorithm. Neuroimage 38, 95-113.

Ashburner, J., and Friston, K. J. (2000). Voxel-based morphometry-the methods. Neuroimage 11, 805-821.

Cheung, S. W., and Larson, P. S. (2010). Tinnitus modulation by deep brain stimulation in locus of caudate neurons (area LC). Neuroscience 169, 1768-1778.

Choi, J. M., Padmala, S., and Pessoa, L. (2011). Impact of state anxiety on the interaction between threat monitoring and cognition. Neuroimage 59, 1912-1923.

Corbetta, M., and Shulman, G. L. (2002). Control of goal-directed and stimulus-driven attention in the brain. Nat. Rev. Neurosci. 3, 201-215.

Corbetta, M., and Shulman, G. L. (2011). Spatial neglect and attention networks. Annu. Rev. Neurosci. 34 569-599.

Craig, A. D. (2003). Interoception: the sense of the physiological condition of the body. Curr. Opin. Neurobiol. 13, 500-505.

DaSilva, A. F., Becerra, L., Pendse, G., Chizh, B., Tully, S., and Borsook, D. (2008). Colocalized structural and functional changes in the cortex of patients with trigeminal neuropathic pain. PLoS One 3:e3396. doi: 10.1371/journal.pone.0003396

Dannlowski, U., Stuhrmann, A., Beutelmann, V., Zwanzger, P., Lenzen, T., Grotegerd, D., Domschke, K., Hohoff, C., Ohrmann, P., Bauer, J., Lindner, C., Postert, C., Konrad, C., Arolt, V., Heindel, W., Suslow, T., and Kugel, H. (2012). Limbic scars: long-term consequences of childhood maltreatment revealed by functional and structural magnetic resonance imaging. Biol. Psychiatry 71, 286-293.

De Ridder, D., Elgoyhen, A. B., Romo, R., and Langguth, B. (2011). Phantom percepts: tinnitus and pain as persisting aversive memory networks. Proc. Natl. Acad. Sci. U.S.A. 108, 8075-8080.

Dobie, R. A. (2003). Depression and tinnitus. Otolaryngol. Clin. North Am. 36, 383-388.

Drevets, W. C., Price, J. L., Simpson, J. R. Jr., Todd, R. D., Reich, T., Vannier, M., and Raichle, M. E. (1997). Subgenual prefrontal cortex abnormalities in mood disorders. Nature 386, 824-827.

Eggermont, J. J., and Roberts, L. E. (2004). The neuroscience of tinnitus. Trends Neurosci. 27, 676-682.

Etkin, A., Egner, T., and Kalisch, R. (2011). Emotional processing in anterior cingulate and medial prefrontal cortex. Trends Cogn. Sci. 15, 85-93.

Eyler, L. T., Prom-Wormley, E., Panizzon, M. S., Kaup, A. R. Fennema-Notestine, C., Neale, M. C., Jernigan, T. L., Fischl, B., Franz, C. E., Lyons, M. J., Grant, M., Stevens, A., Pacheco, J., Perry, M. E., Schmitt, J. E., Seidman, L. J. Thermenos, H. W., Tsuang, M. T., Chen, C. H., Thompson, W. K., Jak, A., Dale, A. M., and Kremen, W. S. (2011). Genetic and environmental contributions to regional cortical surface area in humans: a magnetic resonance imaging twin study. Cereb. Cortex 21, 2313-2321.

Fischl, B., and Dale, A. M. (2000). Measuring the thickness of the human cerebral cortex from magnetic resonance images. Proc. Natl Acad. Sci. U.S.A. 97, 11050-11055.

Fischl, B., Salat, D. H., Busa, E., Albert, M., Dieterich, M., Haselgrove, C., van der Kouwe, A., Killiany, R., Kennedy, D., Klaveness, S., Montillo, A., Makris, N., Rosen, B. and Dale, A. M. (2002). Whole brain segmentation: automated labeling of neuroanatomical structures in the human brain. Neuron 33, 341-355.

Floresco, S. B., Zhang, Y., and Enomoto, T. (2009). Neural circuits subserving behavioral flexibility and their relevance to schizophrenia. Behav. Brain Res. 204, 396-409.

Folmer, R. L., Griest, S. E., Meikle, M. B., and Martin, W. H. (1999). Tinnitus severity, loudness, and depression. Otolaryngol. Head Neck Surg. 121, 48-51.

Fujiwara, E., Schwartz, M. L., Gao, F., Black, S. E., and Levine, B. (2008). Ventral frontal cortex functions and quantified MRI in traumatic brain injury. Neuropsychologia 46, 461-474.

Giraud, A. L., Chery-Croze, S., Fischer, G., Fischer, C., Vighetto, A., Gregoire, M. C., Lavenne, F., and Collet, L. (1999). A selective imaging of tinnitus. Neuroreport 10 , $1-5$.

Goldstein, B., and Shulman, A. (1996). Tinnitus-hyperacusis and the loudness discomfort level test-A preliminary report. Int. Tinnitus J. 2, 83-89.

Good, C. D., Johnsrude, I. S., Ashburner, J., Henson, R. N.,
Friston, K. J., and Frackowiak, R. S. (2001). A voxel-based morphometric study of ageing in 465 normal adult human brains. Neuroimage 14, 21-36.

Griffiths, T. D., and Warren, J. D. (2002). The planum temporale as a computational hub. Trends Neurosci. 25, 348-353.

Gu, J. W., Halpin, C. F., Nam, E. C., Levine, R. A., and Melcher, J. R. (2010). Tinnitus, diminished sound-level tolerance, and elevated auditory activity in humans with clinically normal hearing sensitivity. J. Neurophysiol. 104, 3361-3370.

Hamani, C., Mayberg, H., Stone, S., Laxton, A., Haber, S., and Lozano, A. M. (2011). The subcallosal cingulate gyrus in the context of major depression. Biol. Psychiatry 69, 301-308.

Heller, A. J. (2003). Classification and epidemiology of tinnitus. Otolaryngol. Clin. North Am. 36, 239-248.

Hiller, W., and Goebel, G. (2006). Factors influencing tinnitus loudness and annoyance. Arch. Otolaryngol. Head Neck Surg. 132, 1323-1330.

Husain, F. T., Medina, R. E., Davis, C. W., Szymko-Bennett, Y., Simonyan, K., Pajor, N. M., and Horwitz, B. (2011). Neuroanatomical changes due to hearing loss and chronic tinnitus: a combined VBM and DTI study. Brain Res. 1369, 74-88.

Hutton, C., Draganski, B., Ashburner, J., and Weiskopf, N. (2009). A comparison between voxel-based cortical thickness and voxel-based morphometry in normal aging. Neuroimage 48, 371-380.

Jastreboff, P. J. (1990). Phantom auditory perception (tinnitus): mechanisms of generation and perception. Neurosci. Res. 8 221-254.

Jastreboff, P. J. (2007). Tinnitus retraining therapy. Prog. Brain Res. 166, 415-423.

Kable, J. W., and Glimcher, P. W. (2009). The neurobiology of decision: consensus and controversy. Neuron 63, 733-745.

Koenigs, M., and Grafman, J. (2009). The functional neuroanatomy of depression: distinct roles for ventromedial and dorsolateral prefrontal cortex. Behav. Brain Res. 201, 239-243.

Kroenke, K., Spitzer, R. L., and Williams, J. B. (2001). The PHQ-9: validity of a brief depression severity measure. J. Gen. Intern. Med. 16 606-613.

Kuchinad, A., Schweinhardt, P., Seminowicz, D. A., Wood, P. B.
Chizh, B. A., and Bushnell, M. C. (2007). Accelerated brain gray matter loss in fibromyalgia patients: premature aging of the brain? $J$ Neurosci. 27, 4004-4007.

Landgrebe, M., Langguth, B., Rosengarth, K., Braun, S., Koch, A., Kleinjung, T., May, A., De Ridder, D., and Hajak, G. (2009). Structural brain changes in tinnitus: grey matter decrease in auditory and non-auditory brain areas. Neuroimage 46, 213-218.

Langguth, B., Goodey, R., Azevedo, A., Bjorne, A., Cacace, A., Crocetti, A., Del Bo, L., De Ridder, D., Diges, I., Elbert, T., Flor, H., Herraiz, C., Ganz Sanchez, T., Eichhammer, P., Figueiredo, R., Hajak, G., Kleinjung, T., Landgrebe, M., Londero, A., Lainez, M. J., Mazzoli, M., Meikle, M. B., Melcher, J., Rauschecker, J. P., Sand, P. G., Struve, M., Van de Heyning, P., Van Dijk, P., and Vergara, R. (2007). Consensus for tinnitus patient assessment and treatment outcome measurement: tinnitus research initiative meeting, Regensburg, July 2006. Prog. Brain Res. 166, 525-536.

Leaver, A. M., Renier, L., Chevillet, M. A., Morgan, S., Kim, H. J., and Rauschecker, J. P. (2011) Dysregulation of limbic and auditory networks in tinnitus. Neuron 69, 33-43.

Lemaitre, H., Goldman, A. L., Sambataro, F., Verchinski, B. A., Meyer-Lindenberg, A., Weinberger, D. R., and Mattay, V. S. (2012) Normal age-related brain morphometric changes: nonuniformity across cortical thickness, surface area and gray matter volume? Neurobiol. Aging 33, 617e1-617e9.

Lockwood, A. H., Salvi, R. J., Coad, M. L., Towsley, M. L., Wack, D. S., and Murphy, B. W. (1998). The functional neuroanatomy of tinnitus: evidence for limbic system links and neural plasticity. Neurology 50, 114-120.

Lowry, L. D., Eisenman, L. M., and Saunders, J. C. (2004). An absence of tinnitus. Otol. Neurotol. 25, 474-478.

Lozano, A. M., Mayberg, H. S., Giacobbe, P., Hamani, C., Craddock, R. C., and Kennedy, S. H. (2008). Subcallosal cingulate gyrus deep brain stimulation for treatment-resistant depression. Biol. Psychiatry 64, 461-467.

Mahoney, C. J., Rohrer, J. D., Goll, J. C., Fox, N. C., Rossor, M. N., and Warren, J. D. (2011). Structural neuroanatomy of tinnitus and hyperacusis in semantic dementia. 
J. Neurol. Neurosurg. Psychiatry 82, 1274-1278.

Mattson, A. J., and Levin, H. S. (1990). Frontal lobe dysfunction following closed head injury. A review of the literature. J. Nerv. Ment. Dis. 178, 282-291.

May, A., and Gaser, C. (2006). Magnetic resonance-based morphometry: a window into structural plasticity of the brain. Curr. Opin. Neurol. 19, 407-411.

Mayberg, H. S. (1997). Limbic-cortical dysregulation: a proposed model of depression. J. Neuropsychiatry Clin. Neurosci. 9, 471-481.

Mayberg, H. S., Liotti, M., Brannan, S. K., McGinnis, S., Mahurin, R. K., Jerabek, P. A., Silva, J. A., Tekell, J. L., Martin, C. C., Lancaster, J. L., and Fox, P. T. (1999). Reciprocal limbic-cortical function and negative mood: converging PET findings in depression and normal sadness. Am. J. Psychiatry 156, 675-682.

Mirz, F., Gjedde, A., Ishizu, K., and Pedersen, C. B. (2000). Cortical networks subserving the perception of tinnitus-a PET study. Acta Otolaryngol. 543, 241-243.

Morgan, D. E., Wilson, R. H., and Dirks, D. D. (1974). Loudness discomfort level: selected methods and stimuli. J. Acoust. Soc. Am. 56, 577-581.

Møller, A. R. (2003). Pathophysiology of tinnitus. Otolaryngol. Clin. North Am. 36, 249-266.

Mühlau, M., Rauschecker, J. P., Oestreicher, E., Gaser, C., Röttinger, M., Wohlschläger, A. M., Simon, F., Etgen, T., Conrad, B., and Sander, D. (2006). Structural brain changes in tinnitus. Cereb. Cortex $16,1283-1288$.

Newman, C. W., Jacobson, G. P., and Spitzer, J. B. (1996). Development of the Tinnitus Handicap Inventory. Arch. Otolaryngol. Head Neck Surg. 122, 143-148.

Ongür, D., Ferry, A. T., and Price, J. L. (2003). Architectonic subdivision of the human orbital and medial prefrontal cortex. J. Comp. Neurol. 460, 425-449.
Ongür, D., and Price, J. L. (2000). The organization of networks within the orbital and medial prefrontal cortex of rats, monkeys and humans. Cereb. Cortex 10, 206-219.

Panizzon, M. S., Fennema-Notestine, C., Eyler, L. T., Jernigan, T. L., PromWormley, E., Neale, M., Jacobson, K., Lyons, M. J., Grant, M. D., Franz, C. E., Xian, H., Tsuang, M., Fischl, B., Seidman, L., Dale, A., and Kremen, W. S. (2009). Distinct genetic influences on cortical surface area and cortical thickness. Cereb. Cortex 19, 2728-2735.

Plewnia, C., Reimold, M., Najib, A., Brehm, B., Reischl, G., Plontke, S. K., and Gerloff, C. (2007). Dosedependent attenuation of auditory phantom perception (tinnitus) by PET-guided repetitive transcranial magnetic stimulation. Hum. Brain Mapp. 28, 238-246.

Rauschecker, J. P., Leaver, A. M., and Mühlau, M. (2010). Tuning out the noise: limbic-auditory interactions in tinnitus. Neuron 66, 819-826.

Rauschecker, J. P., and Scott, S. K. (2009). Maps and streams in the auditory cortex: nonhuman primates illuminate human speech processing. Nat. Neurosci. 12, 718-724.

Reyes, S. A., Salvi, R. J., Burkard, R. F., Coad, M. L., Wack, D. S., Galantowicz, P. J., and Lockwood, A. H. (2002). Brain imaging of the effects of lidocaine on tinnitus. Hear. Res. 171, 43-50.

Robinson, S. K., Viirre, E. S., Bailey, K. A., Kindermann, S., Minassian, A. L., Goldin, P. R., Pedrelli, P., Harris, J. P., and McQuaid, J. R. (2008). A randomized controlled trial of cognitive-behavior therapy for tinnitus. Int. Tinnitus J. 14, 119-126.

Salvi, R. J., Wang, J., and Ding, D. (2000). Auditory plasticity and hyperactivity following cochlear damage. Hear. Res. 147, 261-274.

Savitz, J., and Drevets, W. C. (2009). Bipolar and major depressive disorder: neuroimaging the developmental-degenerative divide. Neurosci. Biobehav. Rev. 33, 699-771.
Schecklmann, M., Landgrebe, M., Poeppl, T. B., Kreuzer, P., Männer P., Marienhagen, J., Wack, D S., Kleinjung, T., Hajak, G., and Langguth, B. (2011). Neural correlates of tinnitus duration and Distress: a positron emission tomography study. Hum. Brain Mapp. doi: 10.1002/hbm.21426. [Epub ahead of print]

Schlee, W., Mueller, N., Hartmann, T., Keil, J., Lorenz, I., and Weisz, N. (2009). Mapping cortical hubs in tinnitus. BMC Biol. 7, 80 .

Schneider, P., Andermann, M. Wengenroth, M., Goebel, R., Flor, H., Rupp, A., and Diesch, E. (2009). Reduced volume of Heschl's gyrus in tinnitus. Neuroimage 45 927-939.

Shackman, A. J., Salomons, T. V., Slagter, H. A., Fox, A. S., Winter, J. J., and Davidson, R. J. (2011). The integration of negative affect, pain and cognitive control in the cingulate cortex. Nat. Rev. Neurosci. 12, 154-167.

Shulman, A., Strashun, A. M., Afriyie, M., Aronson, F., Abel, W., and Goldstein, B. (1995). SPECT imaging of brain and tinnitusneurotologic/neurologic implications. Int. Tinnitus J. 1, 13-29.

Spitzer, R. L., Kroenke, K., Williams, J. B., and Lowe, B. (2006). A brief measure for assessing generalized anxiety disorder: the GAD-7. Arch. Intern. Med. 166, 1092-1097.

Steele, J. D., and Lawrie, S. M. (2004) Segregation of cognitive and emotional function in the prefrontal cortex: a stereotactic meta-analysis. Neuroimage 21, 868-875.

Sullivan, M. D., Katon, W., Dobie, R. Sakai, C., Russo, J., and HarropGriffiths, J. (1988). Disabling tinnitus. Association with affective disorder. Gen. Hosp. Psychiatry 10 285-291.

van der Loo, E., Congedo, M., Vanneste, S., De Heyning, P. V., and De Ridder, D. (2011). Insular lateralization in tinnitus distress. Auton. Neurosci. $165,191-194$

Wilson, E. C., Melcher, J. R., Micheyl, C., Gutschalk, A., and Oxenham, A. J. (2007). Cortical FMRI activation to sequences of tones alternating in frequency: relationship to perceived rate and streaming. J. Neurophysiol. 97, 2230-2238.

Winkler, A. M., Kochunov, P., Blangero, J., Almasy, L., Zilles, K., Fox, P. T., Duggirala, R., and Glahn, D. C. (2010). Cortical thickness or grey matter volume? The importance of selecting the phenotype for imaging genetics studies. Neuroimage 53, 1135-1146.

Wong, P. C., Uppunda, A. K., Parrish, T. B., and Dhar, S. (2008). Cortical mechanisms of speech perception in noise. J. Speech Lang. Hear. Res. 51, 1026-1041

Zatorre, R. J., Bouffard, M., Ahad, P., and Belin, P. (2002). Where is "where" in the human auditory cortex? Nat. Neurosci. 5, 905-909.

Zigmond, A. S., and Snaith, R. P. (1983). The hospital anxiety and depression scale. Acta Psychiatr. Scand. 67, 361-370.

Zikopoulos, B., and Barbas, H. (2006). Prefrontal projections to the thalamic reticular nucleus form a unique circuit for attentional mechanisms. J. Neurosci. 26, 7348-7361.

Conflict of Interest Statement: The authors declare that the research was conducted in the absence of any commercial or financial relationships that could be construed as a potential conflict of interest.

Received: 14 January 2012; paper pending published: 24 January 2012; accepted: 16 March 2012; published online: 05 April 2012.

Citation: Leaver AM, Seydell-Greenwald A, Turesky TK, Morgan S, Kim HJ and Rauschecker JP (2012) Cortico-limbic morphology separates tinnitus from tinnitus distress. Front. Syst. Neurosci. 6:21. doi: $10.3389 /$ fnsys.2012.00021

Copyright (c) 2012 Leaver, SeydellGreenwald, Turesky, Morgan, Kim and Rauschecker. This is an open-access article distributed under the terms of the Creative Commons Attribution Non Commercial License, which permits noncommercial use, distribution, and reproduction in other forums, provided the original authors and source are credited. 